\title{
Validation of $\alpha$-Synuclein as a CSF Biomarker for Sporadic Creutzfeldt-Jakob Disease
}

\author{
Franc Llorens ${ }^{1,2} \cdot$ Niels Kruse $^{3} \cdot$ André Karch $^{4} \cdot$ Matthias Schmitz $^{1,2} \cdot$ Saima Zafar $^{1,2}$. \\ Nadine Gotzmann ${ }^{1,2}$ • Ting Sun ${ }^{1,2} \cdot$ Silja Köchy $^{1,2}$ - Tobias Knipper ${ }^{1}$ - Maria Cramm ${ }^{1,2}$. \\ Ewa Golanska $^{5}$ - Beata Sikorska ${ }^{5}$ - Pawel P. Liberski ${ }^{5} \cdot$ Raquel Sánchez-Valle $^{6}$. \\ Andre Fischer ${ }^{2}$ - Brit Mollenhauer ${ }^{3,7,8} \cdot$ Inga Zerr $^{1,2}$
}

Received: 23 December 2016/Accepted: 2 March 2017 /Published online: 21 March 2017

(C) The Author(s) 2017. This article is published with open access at Springerlink.com

\begin{abstract}
The analysis of cerebrospinal fluid (CSF) biomarkers gains importance in the differential diagnosis of prion diseases. However, no single diagnostic tool or combination of them can unequivocally confirm prion disease diagnosis. Electrochemiluminescence (ECL)-based immunoassays have demonstrated to achieve high diagnostic accuracy in a variety of sample types due to their high sensitivity and dynamic range. Quantification of CSF $\alpha$-synuclein (a-syn) by an inhouse ECL-based ELISA assay has been recently reported as an excellent approach for the diagnosis of sporadic Creutzfeldt-Jakob disease (sCJD), the most prevalent form of human prion disease. In the present study, we validated a commercially available ECL-based a-syn ELISA platform as a diagnostic test for correct classification of sCJD cases. CSF a-syn was analysed in $203 \mathrm{sCJD}$ cases with definite diagnosis and in 445 non-CJD cases. We investigated reproducibility and stability of CSF a-syn and made recommendations for
\end{abstract}

its analysis in the sCJD diagnostic workup. A sensitivity of $98 \%$ and a specificity of $97 \%$ were achieved when using an optimal cut-off of $820 \mathrm{pg} / \mathrm{mL}$ a-syn. Moreover, we were able to show a negative correlation between a-syn levels and disease duration suggesting that CSF a-syn may be a good prognostic marker for SCJD patients. The present study validates the use of a-syn as a CSF biomarker of SCJD and establishes the clinical and pre-analytical parameters for its use in differential diagnosis in clinical routine. Additionally, the current test presents some advantages compared to other diagnostic approaches: it is fast, economic, requires minimal amount of CSF and a-syn levels are stable along disease progression.

Keywords Sporadic Creutzfeldt-Jakob $\cdot \alpha$-Synuclein · Cerebrospinal fluid · Biomarkers ·

Electrochemiluminescence $\cdot$ ELISA
Electronic supplementary material The online version of this article (doi:10.1007/s12035-017-0479-5) contains supplementary material, which is available to authorized users.

Franc Llorens

franc.llorens@gmail.com

1 Clinical Dementia Center, Department of Neurology, University Medical Center Göttingen, Robert Koch Stasse 40, 37075 Göttingen, Germany

2 German Center for Neurodegenerative Diseases (DZNE), Site Göttingen, Robert Koch Stasse 40, 37075 Göttingen, Germany

3 Institute for Neuropathology, University Medical Center Göttingen, Göttingen, Germany
4 Department of Epidemiology, Helmholtz Centre for Infection Research, Braunschweig, Germany

5 Department of Molecular Pathology and Neuropathology, Medical University of Lodz, Lodz, Poland

6 Creutzfeldt-Jakob disease unit. Alzheimer's disease and other cognitive disorders unit. Hospital Clínic, Institut d'Investigacions Biomèdiques August Pi i Sunyer, Barcelona, Spain

7 Paracelsus-Elena Klinik, Center for Parkinsonism and Movement Disorders, Kassel, Germany

8 Department of Neurosurgery, University Medical Center Göttingen, Göttingen, Germany 


\section{Introduction}

Creutzfeldt-Jakob disease (CJD) is the most common human prion disease characterised by the accumulation of pathological misfolded prion protein (PrPSc) in the central nervous tissue. Most patients present an unknown aetiology being classified as sporadic Creutzfeldt-Jakob disease (sCJD), which is clinically characterised by the presence of dementia and ataxia $[1,2]$. sCJD affects 1 to 2 per $1,000,000$ individuals per year [3], and it is invariably fatal, usually within 1 year from disease onset [1]. Therefore, biomarkers able to discriminate sCJD from other neurological and neurodegenerative conditions with similar clinical presentation are required $[4,5]$. This is of special importance in the differential diagnostics from alternative forms of rapidly progressive dementias with available treatment to generate an appropriate therapeutic intervention before pathological changes spread throughout the brain [6]. Established CSF biomarkers for SCJD tested in clinical workup in suspected cases are 14-3-3 and total tau (tau). A major limitation of these biomarkers results from the occurrence of their increased levels in acute neurologic disorders and neurodegenerative diseases [7-13]. The clinical diagnosis of SCJD is also supported by the real-time quaking-induced conversion (RT-QuIC) protein aggregation assay, which exhibit a high specificity, and sensitivity similar to the best surrogate biomarkers in detecting SCJD cases [14-17]. However, RT-QuIC presents increased costs, and less inter-laboratory standardisation data is available compared to classical approaches, preventing its widespread implementation in clinical routine.

In this context, electrochemiluminescence-based systems have demonstrated high accuracy, reproducibility, recovery rates and broad dynamic range in the detection of several biomarkers in biological fluids $[18,19]$. Besides its methodological upgrade compared to classical colorimetric platforms, the analytical and clinical performance of electrochemiluminescence technology in the cerebrospinal fluid (CSF) has not been explored in detail in the differential diagnosis of neurodegenerative dementias.

The detection of elevated $\alpha$-synuclein (a-syn) in the CSF of SCJD cases using colorimetric assays was previously reported [20, 21]. However, the differences between sCJD and control groups were rather limited, and the accuracy of the tests was low. Recently, we reported that the use of an in-house a-syn electrochemiluminescence-based enzyme-linked immunosorbent assay (ELISA) platform presented high accuracy in the discrimination of SCJD cases $[19,22]$. However, the implementation of CSF asyn measurements in diagnostic routine of SCJD would get benefit of the validation and establishment of diagnostic accuracy in a commercially available platform, facilitating its technical implementation and inter-laboratory standardisation.
In the present study, we analysed a commercial electrochemiluminescence-based human a-syn ELISA kit as a novel test method in SCJD diagnosis. The present test allowed discriminating non-CJD from SCJD cases with high diagnostic accuracy. Importantly, data were collected from three independent cohorts of SCJD autopsy-confirmed cases. Since standard protocols and sample handling have become more important in routine diagnostic, recommendations for the CSF a-syn diagnostic of SCJD are reported based on the study of the influence of pre-analytical and analytical parameters.

\section{Methods}

\section{Subjects}

The study included a total of 648 patients recruited at the National Reference Center for Transmissible Spongiform Encephalopathies of the Department of Neurology and Neuropathology at the University Medical Center of Gottingen, Germany (cohort 1), at Polish neurologic and psychiatric hospital departments (cohort 2) and at the Unit of Biodiagnostic of CJD and other prion diseases at the Hospital Clinical of Barcelona, Spain (cohort 3). All patients with SCJD were classified as definite cases by neuropathological examination according to diagnostic consensus criteria [23]. Non-CJD cases were composed of patients referred to the same centres where prion disease diagnosis was excluded according to clinical criteria or autopsy. Non-CJD cases included the following: neurologically healthy patients (no neurological clinical diagnosis and normal neuro-physicological assessment) $(n=41)$ and patients suffering from neurological diseases $(n=149)$ or neurodegenerative conditions and cognitive impairment/dementia $(n=255)$. Neurological diseases included psychiatric disorders (psychosis, bipolar disorder, depression and schizophrenia), ischemic stroke, epilepsy, autoimmune diseases, meningitis, alcohol abuse, headache, vertigo, pain syndromes, acute hypoxia, encephalopathy, cerebral vasculitis, normal pressure hydrocephalus and alternative neurologic conditions. Neurodegenerative disease included Parkinson's disease, dementia with Lewy bodies, multiple system atrophy, Alzheimer's disease, frontotemporal dementia, Huntington's disease, vascular dementia, corticobasal degeneration, progressive supranuclear palsy and olivopontocerebellar atrophy.

CSF samples were tested for blood contamination using Hemastix strips (Siemens), and cases containing more than 25 erythrocytes $/ \mathrm{mm}^{3}$ and/or haemoglobin contamination were intentionally excluded from this study in order to avoid possible false positive outcomes. Information about demographics and CSF biomarkers tau and 14-3-3 of the study population are supplied in Table 1. 
Table 1 Demographic characteristics of the study population analysed and CSF prion biomarker data

\begin{tabular}{lll} 
Demographics & non-CJD & $s C J D$ \\
Number of cases & 445 & 203 \\
Age (mean \pm SD (years)) & $66 \pm 12$ & $66 \pm 9$ \\
Gender (w/m in \%) & $54 / 46$ & $52 / 48$ \\
sCJD biomarkers & & \\
14-3-3 (p-w-n in \%) & $12-2-84$ & $91-2-7$ \\
tau (mean $\pm \mathrm{SD}(\mathrm{pg} / \mathrm{mL}))$ & $402 \pm 376$ & $5965 \pm 4911$ \\
b & & \\
$\alpha$-synuclein (pg/mL) & $n o n-C J D$ & $s C J D$ \\
Mean & 324 & 8906 \\
Standard deviation & 214 & 7790 \\
Standard error & 10 & 548 \\
Minimum & 70 & 513 \\
25\% percentile & 200 & 3183 \\
Median & 259 & 6595 \\
$75 \%$ percentile & 364 & 11,950 \\
Maximum & 1908 & 41,960 \\
Lower 95\% CI & 304 & 7828 \\
Upper 95\% CI & 344 & 9984 \\
\hline
\end{tabular}

a. The total number of cases, age (mean $\pm \mathrm{SD}$ in years) and gender distribution as well as tau (mean $\pm \mathrm{SD}$ in $\mathrm{pg} / \mathrm{mL}$ ) and 14-3-3 semiquantification (\% of positive (p), weak/inconclusive (w) and negative (n)) is reported. b. CSF a-syn levels (in $\mathrm{pg} / \mathrm{mL}$ ) in non-CJD and sCJD cases. Mean values, standard deviation and standard error, percentile values and lower and upper $95 \% \mathrm{CI}$ are indicated

\section{Statistical Methods}

Mann-Whitney $U$ tests were used to compare two groups of samples. For analysis of a-syn stability, Wilcoxon signed rank sum tests were applied. In order to assess the diagnostic accuracy of a-syn, receiver operating characteristic (ROC) curve analyses were carried out and areas under the curve (AUC) with 95\% confidence intervals were calculated using GraphPad Prism 6.01. The best cut-off value was estimated based on the Youden index [24] derived from cohorts 2 and 3 and was then externally validated in cohort 1 . To compare the sensitivities between biomarkers, the McNemar's test was used. Spearman rank correlation was used to test association between biomarker levels. For codon 129 polymorphism comparison, cases were tested for normality, and Kruskal-Wallis test followed by Dunns post-test was applied. In order to determine the effect of a-syn levels on clinical outcome, the association between a-syn levels and total disease duration (time between disease onset and death) was assessed using a fractional polynomial approach.

\section{CSF Analysis}

Quantification of a-syn was performed using the electrochemiluminescence-based ELISA-based human a-syn kit from Meso Scale Discovery (MSD) (Catalogue No. K151TGD) following manufacturer's instructions. Briefly, assay plates were blocked by adding $150 \mu \mathrm{l}$ of Diluent 35 to each well. Plates were sealed and incubated at room temperature with shaking at $300 \mathrm{rpm}$ for $1 \mathrm{~h}$. Then, plates were washed three times with $150 \mu \mathrm{l}$ per well of PBS-T (PBS supplemented with $0.05 \%$ Tween-20). Detection antibody $(25 \mu \mathrm{l})$ and CSF (diluted 1:8) or calibrator $(25 \mu \mathrm{l})$ was added to each well. Plates were sealed and incubated at room temperature with shaking at $700 \mathrm{rpm}$ for $2 \mathrm{~h}$. After washing as indicated above, $150 \mu \mathrm{l}$ of $2 \times$ read buffer $\mathrm{T}$ was added to each well. Measurements were performed on a MSD Sector Imager 6000. Calibrators were run in duplicate to generate calibration curve.

Tau was quantified using the INNOTESThTAU-Ag (Fujirebio, Gent, Belgium) ELISA test. 14-3-3 was semiquantitatively tested by using Western blot as previously described [8].

\section{Analysis of a-syn Stability}

To evaluate the effects of CSF storage conditions (temperature and freezing/thawing cycles) on a-syn concentration, CSF samples from $8 \mathrm{sCJD}$ patients were stored in polypropylene tubes at room temperature and $4{ }^{\circ} \mathrm{C}$ for 1,3 and 6 days. In addition, CSF samples were subjected to 2,4 and 8 repeated freezing and thawing cycles. a-syn concentrations at each time point or cycle were calculated as percent of control (time point zero), which was defined as $100 \%$.

\section{Ethics}

The study was conducted according to the revised Declaration of Helsinki and Good Clinical Practice guidelines and approved by local Ethics committees Goettingen (No. 9/6/08, 19/11/09, 18/8/15). Informed consent was given by all study participants or their legal next of kin.

\section{Results}

\section{ELISA Test Performance in the Quantification of CSF a-syn Quantification}

CSF a-syn levels were measured using the electrochemilu ]minescence-based human a-syn kit (Fig. 1a). The dynamic range of the assay was from $1.52 \pm 0.42$ to $10,000 \mathrm{pg} / \mathrm{mL}$ asyn. The precision of the assay was validated by the low coefficients of variation (CV) measured in separate runs, 


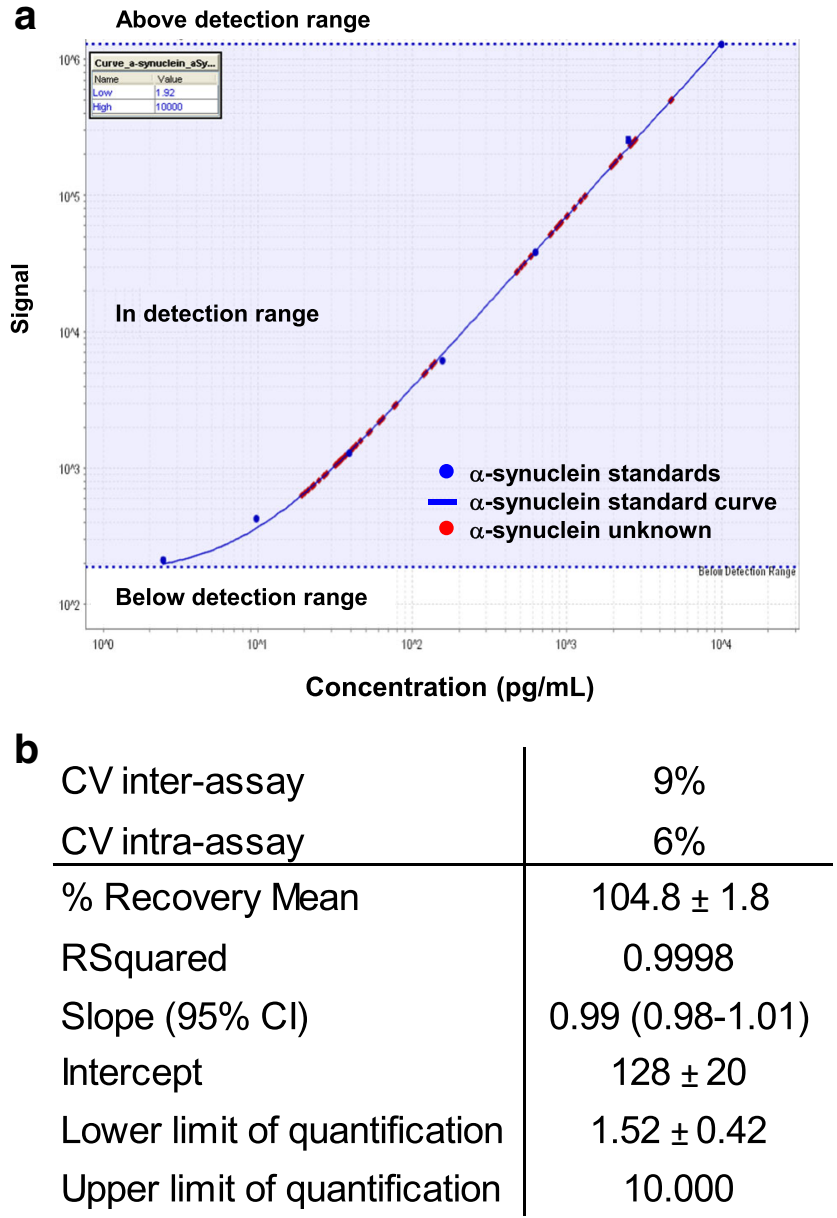

Fig. 1 Quantification of CSF a-syn by the human ELISA kit from MSD. a Representative plot for quantification of CSF a-syn in samples from CJD and non-CJD cases. The dynamic range is indicated in blue. Calibrators are denoted as blue spots and unknown samples as red spots. CSF samples from diverse aetiology were in detection range using a 1:8 dilution. b Analytical and technical parameters derived from quantification of CSF a-syn with the human ELISA kit from MSD are shown

samples, laboratories and analysers as suggested elsewhere [25]. In all cases, repeatability and within-lab/plate reproducibility were below $9 \%$ (Fig. 1b), validating precision and limits of quantification of the assay. Inter-lot and interlaboratory variability were lower than 11 and $12 \%$, respectively. Dilution linearity was assessed in serial dilutions of CSF to avoid hook effect. Selected CSF dilution (1:8) permitted the lineal quantification of the lowest and highest a-syn levels without the need to analyse multiple sample dilutions.

\section{Diagnostic Accuracy of CSF a-syn as sCJD Biomarker}

Our study cohort included a total of 445 non-CJD and 203 sCJD cases. CSF a-syn showed an excellent accuracy in discriminating non-CJD from sCJD cases $(p<0.001$ and $\mathrm{AUC}=0.997,95 \%$ CI: 0.995 to 0.999 ) (Fig. 2a, b). Mean a-

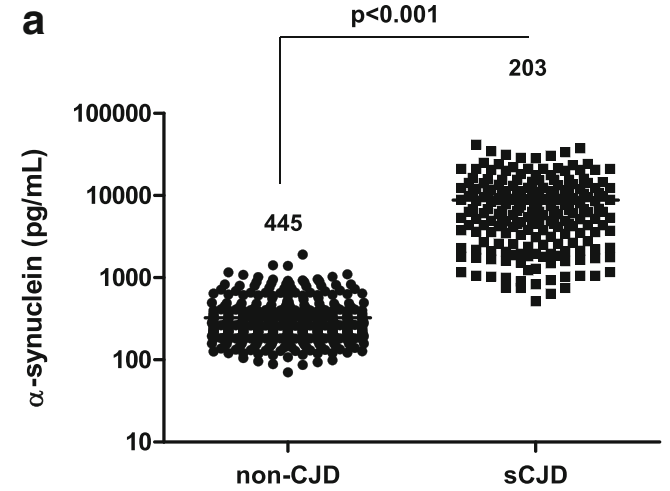

b

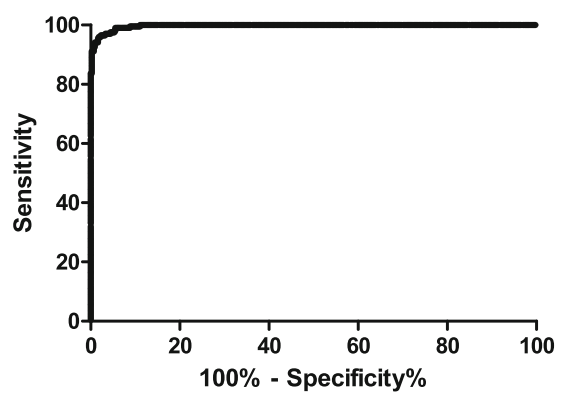

\begin{tabular}{lc}
\multicolumn{2}{l}{ Area under the $R$ OC curve } \\
\hline \hline Area & 0.9970 \\
Standard Error & 0.0011 \\
$95 \% \mathrm{Cl}$ & 0.9948 to 0.9991 \\
$\mathrm{P}$ value & $<0.0001$
\end{tabular}

\begin{tabular}{lc} 
Clinical accuracy & \\
\hline \hline Sensitivity & $98 \%(95 \% \mathrm{Cl}: 93-99 \%)$ \\
Specificity & $97 \%(95 \% \mathrm{Cl}: 95-99 \%)$ \\
Cut-off & $820 \mathrm{pg} / \mathrm{mL}$
\end{tabular}

Fig. 2 Diagnostic accuracy of CSF a-syn levels as SCJD biomarker. a CSF a-syn levels in non-sCJD and sCJD. Statistically significant differences were detected between non-CJD and SCJD cases $(p<0.001)$. b ROC curve for a-syn in the comparative analysis between non-sCJD cases and SCJD cases. Sensitivity and specificity, receiver operating characteristic (ROC) curves and derived area under the curve (AUC) were calculated. Based on Youden Index, an optimal cut-off of $820 \mathrm{pg} / \mathrm{mL}$ a-syn and a sensitivity of $98 \%$ and specificity of $97 \%$ were achieved for the discrimination of sCJD from non-CJD cases

syn values were $324 \pm 214$ in non-CJD and $8906 \pm 7790$ in sCJD (Table 1b). Using a cut-off of $820 \mathrm{pg} / \mathrm{mL}$ of a-syn (optimal level identified by Youden index in the training cohorts), a sensitivity of $98 \%$ (95\% CI: 93-99\%) and a specificity of 97\% (95\% CI: 95-99\%) was achieved in the validation cohort (Fig. 2b). Since high sensitivities are reported for CSF 14-3-3 and tau tests in the detection of SCJD cases [7-13, 26, 27] (Supplementary Table 2), we aimed to compare the performance of the three biomarkers in the same population. 14-33 and tau from cohort $1(n=128)$ presented sensitivities of 91 
and $92 \%$, respectively, in detecting SCJD cases, which was significantly lower than those obtained for CSF a-syn (98\%). Neither a specific diagnostic group nor a correlation between a-syn and tau/14-3-3 profile was detected in the non-CJD cases tested positive for a-syn. Comparison of sensitivities in cohort 1 between the three biomarkers revealed statistical differences between a-syn vs tau $(p=0.035)$ and a-syn vs 14-3-3 $(p=0.021)$ comparisons, but not in tau vs 14-3-3 ( $p=0.763)$. Additionally, we found a positive correlation between CSF asyn and tau levels $(p<0.001)$ in control subjects and in SCJD cases, in agreement with the observations from our previous study [22]. Next, we investigated the role of $P R N P$ codon 129 polymorphism on CSF a-syn levels. Information about codon polymorphism was available for 182 cases $(\mathrm{MM}=120$, $\mathrm{MV}=23$ and $\mathrm{VV}=34$ ). As we previously reported [22], asyn levels in MV cases $(5391 \pm 4780 \mathrm{pg} / \mathrm{mL}$ a-syn) were significantly lower than those detected in MM (9695 $\pm 7605 \mathrm{pg} / \mathrm{mL}$ a-syn) $(p<0.05)$. Mean values in MV cases were also lower than in VV $(9941 \pm 9733 \mathrm{pg} / \mathrm{mL}$ a-syn $)$. However, these differences were not statistically significant.

\section{Influence of Timing in CSF Tests and of Disease Duration}

Next, we assessed the influence of time of CSF sampling on asyn concentration in the diagnosis of SCJD. For this, samples from $10 \mathrm{sCJD}$ patients for which CSF at different stages of disease was available were tested. Since disease duration in SCJD is highly variable, depending on demographic and genetic characteristics [2], the time interval between disease onset and lumbar puncture (LP) is not an appropriate estimate of the stage of disease when the LP was performed. Instead, we divided the time of LP to disease onset in each patient by the total duration of the disease. Then, samples were grouped in three categories according to whether they underwent LP in the first (time of LP to disease onset/total duration of the disease $<0.33)$, second $(0.33-0.66)$, or third $(>0.66)$ stage of the disease, as previously reported [28]. No major alterations in CSF a-syn concentration were detected between different LPs in nine of the cases (Fig. 3a). One case presented a decrease in a-syn levels, most likely related to its rapid disease course ( 3 weeks from disease onset to death).

CSF a-syn levels were inversely associated with disease duration $(p<0.001)$ in the 185 cases where disease duration was known (Fig. 3b). This suggests a role for CSF a-syn as a prognostic SCJD marker. In this regard, our data indicate that when a-syn levels are higher than $20,000 \mathrm{pg} / \mathrm{mL}$, disease duration was inevitably shorter than 1 year. By using fractional polynomial linear regression analysis, the expected duration of disease from the a-syn value can be estimated by using the following equation: duration in months $=5.70+1.43 *((\mathrm{a}-\mathrm{syn}$ $\left.(\mathrm{pg} / \mathrm{mL}) / 10,000)^{-0.5}-0.99\right)-0.57 *((\mathrm{a}-\mathrm{syn}(\mathrm{pg} / \mathrm{mL}) / 10,000)-$ 1.02).
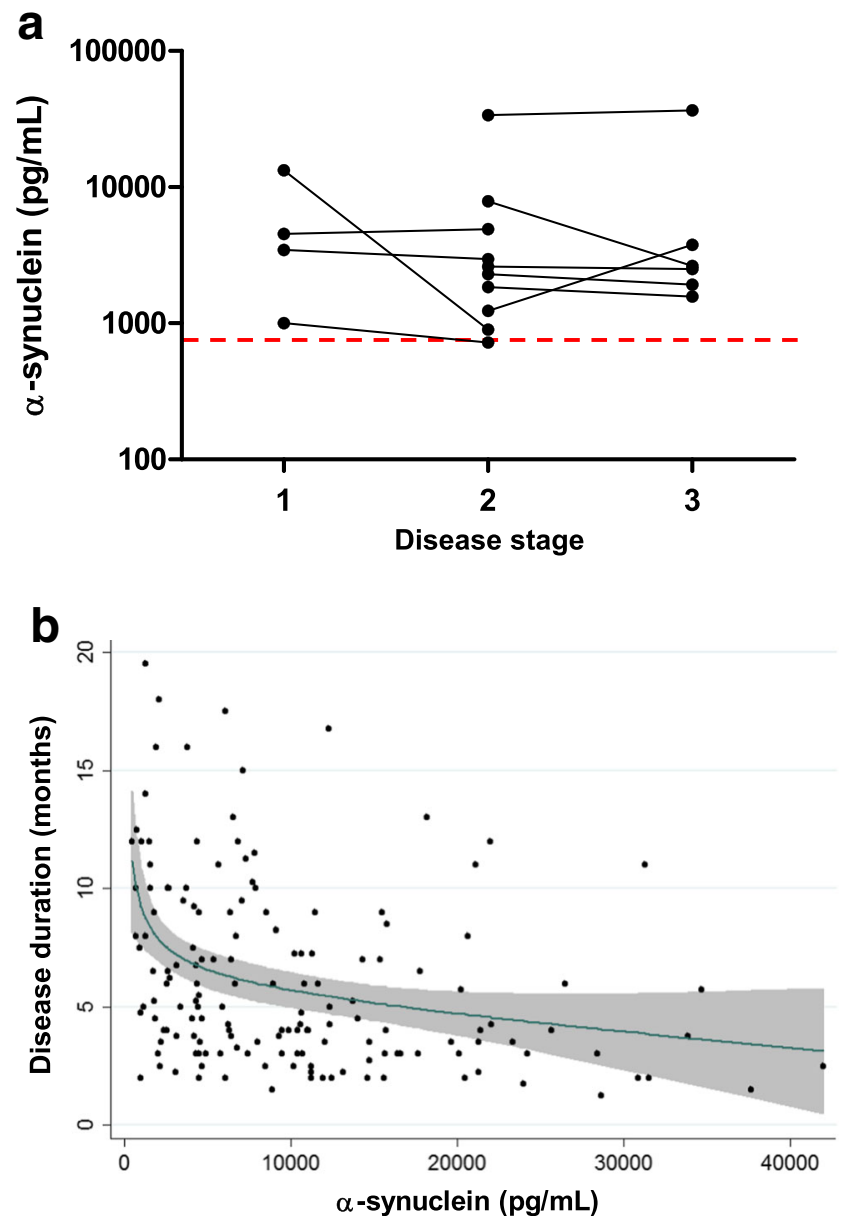

Fig. 3 Influence of timing and disease duration in CSF a-syn levels. a CSF a-syn levels in serial LPs in SCJD cases at different stages of the disease. Samples were grouped in three categories according to whether they underwent LP in the first (time from disease onset to LP/total duration of the disease $<0.33)$, second $(0.33-0.66)$ or third $(>0.66)$ stage of the disease. Dashed line indicates established cut-off for sCJD. b Association between CSF a-syn levels and disease duration (months) in SCJD patients analysed by a fractional polynomial linear regression approach $(p<0.001)$. Displayed is the functional form of the association with $95 \%$ confidence intervals. The expected duration of disease can be estimated by using the following equation: duration in months $=5.70+1.43 *\left((\mathrm{a}-\mathrm{syn}(\mathrm{pg} / \mathrm{mL}) / 10,000)^{-0.5}-0.99\right)-0.57 *((\mathrm{a}-\mathrm{syn}$ $(\mathrm{pg} / \mathrm{mL}) / 10,000)-1.02)$

\section{Effect of Multiple Freeze/Thaw Cycles and Defined Storage Conditions}

Storage of CSF at room temperature or at $4{ }^{\circ} \mathrm{C}$ for up to 6 days had no effect on a-syn concentration (Fig. 4a, b). CSF a-syn was stable for up to four freezing/thawing cycles while a decrease of $9.7 \pm 6.7 \%(p<0.05)$ was detected after 8 cycles (Fig. 4c). Additionally, CSF storage for 12 months at $-80{ }^{\circ} \mathrm{C}$ did not alter the levels of a-syn in a subset of 3 non-CJD and 3 sCJD cases (data not shown). 

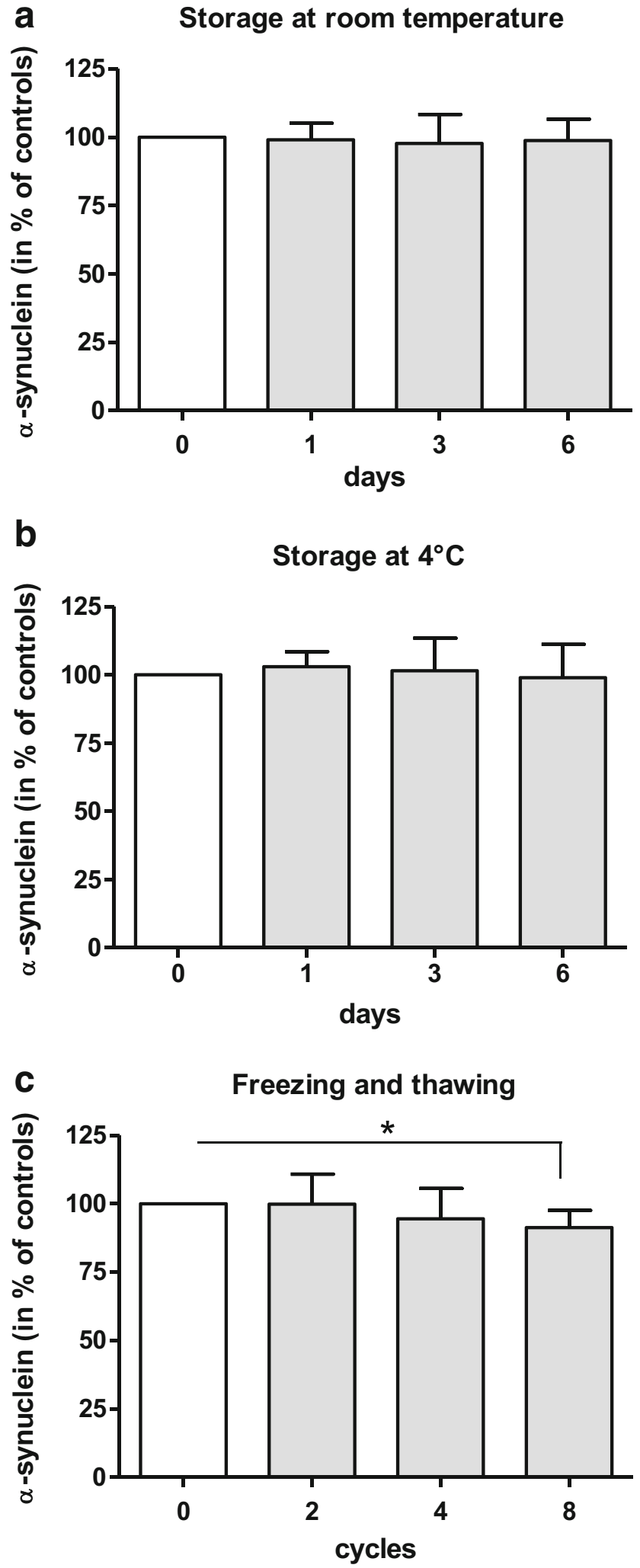

\section{Discussion}

CSF a-syn quantification by an in-house electrochemilu minescence-based ELISA platform has been recently reported
Fig. 4 Effect of short- and long-term storage on CSF a-syn concentration in SCJD patients. CSF a-syn levels were determined as indicated in "Material and Methods". CSF a-syn concentration is shown relative to the reference sample (time point 0 ), which was set as $100 \%$. The concentration of CSF a-syn from 8 different SCJD patients $(n=8)$ was determined $\mathbf{a}$ before and after storage at room temperature ( 0 to 6 days), $\mathbf{b}$ storage at $4{ }^{\circ} \mathrm{C}$ ( 0 to 6 days) and $\mathbf{c}$ after repeated freezing and thawing cycles ( 0 to 8 cycles). A significant loss of CSF a-syn level could be observed in samples subjected to 8 freezing-thawing cycles. Error bars represent standard deviations $(\mathrm{SD})$

as a useful biomarker in the discrimination of SCJD cases from a broad range of neurological and neurodegenerative disorders [22]. However, implementation of the assay in clinical diagnostics requires the following: (i) the validation and standardisation of a commercially available kit, along with definition of diagnostic accuracy and cut-off values, and (ii) investigation of relevant pre-analytical parameters to be considered for sample collection and management. In the present study, the human a-syn kit from Meso Scale Discovery® has been validated as a diagnostic test for SCJD. Clinical accuracy has been established, and pre-analytical parameters for appropriate sample handling are reported. The ELISA kit showed high reproducibility (intra- and inter-assays $<9 \%$ ) and sensitivity and a broad dynamic range. The wide dynamic range of the detection system ( $4 \operatorname{logs}$ ) allowed the quantification of asyn levels without the need of multiple sample dilutions. Because of the high sensitivity of the technology, lower amounts of CSF are required compared to other approaches. Other advantages of the present system include its low background since stimulation method (electricity) is coupled to the signal (light) allowing only labels near the electrode surface to be detected.

In the study of the accuracy of a-syn levels as SCJD biomarker, we analysed one of the largest studied cohorts with definite prion disease. At the optimal cut-off of $820 \mathrm{pg} / \mathrm{mL}$ asyn, we obtained a specificity of $98 \%$ and a sensitivity of $97 \%$ in the validation cohort. Importantly, biomarker cut-off was externally validated in an independent cohort from a different country, underlining the relevance of the findings.

Overall, a-syn test shows an optimal diagnostic accuracy in the specific detection of SCJD cases, with clinical parameters in the range of the best currently available prion biomarkers (Supplementary Table 2) such as p-tau/tau ratio [29] and the newly implemented real-time quaking-induced conversion RT-QuIC [15]. Additionally, the present ELISA tests displayed a similar analytical performance as previously reported for an in-house electrochemiluminescence-based ELISA platform [22], reinforcing the robustness of the method.

Several CSF biomarkers are currently used as valuable tools for the diagnosis of sCJD. While most of them are surrogate disease markers (14-3-3, tau, S100B, NSE) [7], RTQuIC directly measures the amount of pathogenic prion 
protein able to induce the conversion of recombinant PrP molecules in a fluorimetric seeding assay [15-17]. Surrogate markers with highest sensitivity such as 14-3-3 and tau present lower specificity when tested on several neurological conditions [7, 29, 30]. Indeed, positive 14-3-3 signal can be detected in the CSF of various neurological disorders such as acute stroke, meningoencephalitis, and subarachnoidal haemorrhage [31,32], while elevated tau is a common hallmark in several types of neurodegenerative dementias (Alzheimer's disease, mild cognitive impairment, vascular dementia, and fronto-temporal dementia) [29,33] and in acute ischemic events [34]. On the contrary, elevated a-syn is not detectable in any group of neurological and neurodegenerative disorders as reported before [22], and in the present study, enhancing the specificity of the test over other biomarker approaches. In this regard, RT-QuIC overcomes the handicap of surrogate biomarkers regarding specificity; however, sensitivity is still not optimal as shown in several other studies (85-96\%) [16]. Furthermore, a-syn measurement is faster $(3.5 \mathrm{~h})$ than for first and second generation RT-QuIC assays ( $<2$ days and 4-14 h, respectively) [15, 17]. Regarding pre-analytical conditions, some inconsistencies are reported in the bibliography. Regarding pre-analytical conditions, some inconsistencies are reported in the bibliography. While CSF a-syn levels were found to be decreased after 4 days at $4{ }^{\circ} \mathrm{C}$ and 6 freeze/thaw cycles [35], Kruse et al., in agreement with our observations, showed a great stability on a-syn levels on a broad range of pre-analytical conditions, including freeze/thawing cycles and temperature storage conditions [36]. A limitation of CSF asyn quantification is the increased abundance of a-syn in peripheral blood [37]; thus, CSF samples must be tested for blood contamination in relation to this test. Indeed, presence of blood contamination is usually tested in prion diagnosis routine due to its influence in other CSF biomarkers such as 14-3-3 and RT-QuIC [17, 27]. Although new generation ELISA platforms are not yet implemented in most clinical routine laboratories, their reported technical advantages over classical colorimetric systems and the possibility to simultaneously test different analytes in a single run will help to increase its widespread implementation in dementia diagnostic work up.

SCJD disease duration varies depending on demographic and genetic factors from several weeks to 3 years [3], with an average disease duration of 5 months, and only $14 \%$ of the patients surviving longer than 1 year (Creutzfeldt-Jakob Disease International Surveillance Network, http://www. eurocjd.ed.ac.uk). Here, we also demonstrated that CSF asyn levels are stable along disease duration as reported in a group of SCJD cases ranging from the lowest to the highest asyn levels for which serial LPs were available. Altogether, our study supports the use of CSF a-syn quantification in diagnostic routine as a first robust, cheap and fast discriminatory assay to detect SCJD cases, which could be further validated by
Table 2 Recommendations for standardisation and pre-analytical treatment of CSF samples for the analysis of a-syn in SCJD diagnostics

\begin{tabular}{ll}
\hline 1 Tube selection & Polypropylene tubes \\
2. CSF volume & $0.5-1.5 \mathrm{~mL}$ \\
3. Shipping time & Up to 6 days at $4{ }^{\circ} \mathrm{C}$ or room temperature \\
4. After arrival & Specimen macroscopically clear \\
& Assessment for blood contamination inspection \\
& supplemented with hemasticks or routine \\
& microscopic quantification \\
& Centrifugation at $1500 \mathrm{xg}$ for 10 min \\
& Freeze at $-80{ }^{\circ} \mathrm{C}$ until test is performed \\
& Stable for at least 6 cycles \\
5. Freeze/thaw & Stable for at least 12 months at $-80{ }^{\circ} \mathrm{C}$ \\
6. Long-term storage
\end{tabular}

means of RT-QuIC test to achieve a defined pre-mortem diagnosis.

We summarised the outcome of our study and prepared a recommendation table for pre-analytical sample handling and storage to improve standardisation of CSF a-syn quantification for the diagnosis of SCJD patients in clinical routine (Table 2). See also recommendations for sample handling published recently [35].

A potential further application of CSF a-syn quantification is its use as prognostic marker, since disease duration showed a negative correlation with a-syn levels, comparable to CSF tau [38]. In this regard, a valuable outcome of our work is the presentation of an equation to estimate the expected duration of disease from the a-syn value. Indeed, identification of genetic, demographic and biochemical predictors of survival time is of crucial interest, not only for diagnosis and counselling, but also for the correct assessment and evaluation of an eventual therapeutic intervention.

$a$-syn, $\alpha$-synuclein; $A U C$, area under the curve; $C J D$, Creutzfeldt-Jakob disease; $E C L$, Electrochemiluminescence; ELISA, enzyme-linked immunosorbent assay; $L P$, lumbar puncture; $C S F$, cerebrospinal fluid; $P r P S c$, pathological misfolded prion protein; $R O C$, receiver operating characteristic; RT-QuIC, real-time quaking-induced conversion; $s C J D$, sporadic Creutzfeldt-Jakob disease; SD, standard deviation.

Acknowledgements This study was supported by a grant from the European Commission: PRIORITY FP7 (grant no. 222887), by the EU joint programme-Neurodegenerative Disease Research (JPNDDEMTEST: Biomarker based diagnosis of rapidly progressive dementias-optimization of diagnostic protocols, 01ED1201A), by the Robert-Koch Institute thought the Bundesministerium für Gesundheit (NRZ 1369-341) and by the Federal Ministry of Education and Research grants within the German Network for Degenerative Dementia to IZ, by the German Center for Neurodegenerative Diseases (DZNE) to AF, and by the Red Nacional de Priones (AGL2015-71764REDT-MINECO) to FL, IZ and RSV. The funding bodies had no role in study design and collection, analysis and interpretation of data. 
Authors' Contributions FL and IZ conceived the study, FL, NK, NG, TS and SK performed analysis, FL, NK, AK, MS and MC analysed data, TK, EG, BS, PPL, RSV, AF, BM and IZ characterised patients and/or contributed samples and technical expertise. SZ critically reviewed the manuscript. FL drafted the manuscript. All authors interpreted the data, revised the manuscript for important intellectual content and read and approved the final manuscript version.

\section{Compliance with Ethical Standards}

Competing Interests The authors declare that they have no competing interests.

Open Access This article is distributed under the terms of the Creative Commons Attribution 4.0 International License (http:// creativecommons.org/licenses/by/4.0/), which permits unrestricted use, distribution, and reproduction in any medium, provided you give appropriate credit to the original author(s) and the source, provide a link to the Creative Commons license, and indicate if changes were made.

\section{References}

1. Puoti G, Bizzi A, Forloni G et al (2012) Sporadic human prion diseases: molecular insights and diagnosis. Lancet Neurol 11: 618-628. doi:10.1016/S1474-4422(12)70063-7

2. Gambetti P, Kong Q, Zou W et al (2003) Sporadic and familial CJD: classification and characterisation. Br Med Bull 66:213-239. doi: 10.1093/bmb/66.1.213

3. Heinemann U, Krasnianski A, Meissner B et al (2007) CreutzfeldtJakob disease in Germany: a prospective 12-year surveillance. Brain 130:1350-1359. doi:10.1093/brain/awm063

4. Geschwind MD (2016) Rapidly progressive dementia. Contin Lifelong Learn Neurol 22:510-537. doi:10.1212/CON. 0000000000000319

5. Schmidt C, Wolff M, Weitz M et al (2011) Rapidly progressive Alzheimer disease. Arch Neurol 68:1124-1130. doi:10.1001/ archneurol.2011.189

6. Paterson RW, Takada LT, Geschwind MD (2012) Diagnosis and treatment of rapidly progressive dementias. Neurol Clin Pract 2: 187-200. doi:10.1212/CPJ.0b013e31826b2ae8

7. Sanchez-Juan P, Green A, Ladogana A et al (2006) CSF tests in the differential diagnosis of Creutzfeldt-Jakob disease. Neurology 67: 637-643. doi:10.1212/01.wnl.0000230159.67128.00

8. Zerr I, Bodemer M, Gefeller O et al (1998) Detection of 14-3-3 protein in the cerebrospinal fluid supports the diagnosis of Creutzfeldt-Jakob disease. Ann Neurol 43:32-40. doi:10.1002/ ana.410430109

9. Stoeck K, Sanchez-Juan P, Gawinecka J et al (2012) Cerebrospinal fluid biomarker supported diagnosis of Creutzfeldt-Jakob disease and rapid dementias: a longitudinal multicentre study over 10 years. Brain 135:3051-3061. doi:10.1093/brain/aws238

10. Otto M, Wiltfang J, Cepek L et al (2002) Tau protein and 14-3-3 protein in the differential diagnosis of Creutzfeldt-Jakob disease. Neurology 58:192-197. doi:10.1212/WNL.58.2.192

11. Chohan G, Pennington C, Mackenzie JM et al (2010) The role of cerebrospinal fluid 14-3-3 and other proteins in the diagnosis of sporadic Creutzfeldt-Jakob disease in the UK: a 10-year review. J Neurol Neurosurg Psychiatry 81:1243-1248. doi:10.1136/jnnp. 2009.197962

12. Leitão MJ, Baldeiras I, Almeida MR et al (2016) Sporadic Creutzfeldt-Jakob disease diagnostic accuracy is improved by a new CSF ELISA 14-3-3?? Assay. Neuroscience 322:398-407. doi:10.1016/j.neuroscience.2016.02.057
13. Coulthart MB, Jansen GH, Olsen E et al (2011) Diagnostic accuracy of cerebrospinal fluid protein markers for sporadic CreutzfeldtJakob disease in Canada: a 6-year prospective study. BMC Neurol 11:133. doi:10.1186/1471-2377-11-133

14. Atarashi R, Satoh K, Sano K, Fuse T, Yamaguchi N, Ishibashi D, Matsubara T, Nakagaki T, Yamanaka H, Shirabe S, Yamada M, Mizusawa H, Kitamoto T, Klug G, McGlade A, Collins SJ, Nishida N (2011) Ultrasensitive human prion detection in cerebrospinal fluid by real-time quaking-induced conversion. Nat Med 17(2):175-8

15. Orru CD, Groveman BR, Hughson AG et al (2015) Rapid and sensitive RT-QuIC detection of human Creutzfeldt-Jakob disease using cerebrospinal fluid. MBio. doi:10.1128/mBio.02451-14

16. McGuire LI, Peden AH, Orru CD et al (2012) Real time quakinginduced conversion analysis of cerebrospinal fluid in sporadic Creutzfeldt-Jakob disease. Ann Neurol 72:278-285. doi:10.1002/ ana.23589

17. Cramm M, Schmitz M, Karch A et al (2016) Stability and reproducibility underscore utility of RT-QuIC for diagnosis of Creutzfeldt-Jakob disease. Mol Neurobiol 53:1896-1904. doi:10. 1007/s12035-015-9133-2

18. Pan C, Korff A, Galasko D et al (2015) Diagnostic values of cerebrospinal fluid T-tau and A $\beta 42$ using Meso scale discovery assays for Alzheimer's disease. J Alzheimers Dis 45:709-719. doi:10. 3233/JAD-143099

19. Llorens F, Kruse N, Schmitz M et al (2015a) Quantification of CSF biomarkers using an electrochemiluminescence-based detection system in the differential diagnosis of $\mathrm{AD}$ and $\mathrm{sCJD}$. J Neurol 262:2305-2311. doi:10.1007/s00415-015-7837-x

20. Kasai $T$, Tokuda $T$, Ishii $R$ et al (2014) Increased $\$ \alpha \$$-synuclein levels in the cerebrospinal fluid of patients with Creutzfeldt-Jakob disease. J Neurol 261:1203-1209. doi:10.1007/s00415-014-7334-7

21. Llorens F, Zafar S, Ansoleaga B et al (2015b) Subtype and regional regulation of prion biomarkers in sporadic Creutzfeldt-Jakob disease. Neuropathol Appl Neurobiol 41:631-645. doi:10.1111/nan. 12175

22. Llorens F, Kruse N, Schmitz M et al (2016) Evaluation of $\alpha$ synuclein as a novel cerebrospinal fluid biomarker in different forms of prion diseases. Alzheimers Dement:1-10. doi:10.1016/j. jalz.2016.09.013

23. Parchi P, De Boni L, Saverioni D et al (2012) Consensus classification of human prion disease histotypes allows reliable identification of molecular subtypes: an inter-rater study among surveillance centres in Europe and USA. Acta Neuropathol 124:517-529. doi: 10.1007/s00401-012-1002-8

24. Youden WJ (1950) Index for rating diagnostic tests. Cancer 3:3235. doi: $10.1002 / 1097-0142(1950) 3: 1<32:$ :A ID CNCR2820030106>3.0.CO;2-3

25. Andreasson U, Perret-Liaudet A, van Waalwijk van Doorn LJC et al (2015) A practical guide to immunoassay method validation. Front Neurol. doi:10.3389/fneur.2015.00179

26. Matsui Y, Satoh K, Miyazaki T et al (2011) High sensitivity of an ELISA kit for detection of the gamma-isoform of 14-3-3 proteins: usefulness in laboratory diagnosis of human prion disease. BMC Neurol 11:120. doi:10.1186/1471-2377-11-120

27. Schmitz M, Ebert E, Stoeck K et al (2015) Validation of 14-3-3 protein as a marker in sporadic Creutzfeldt-Jakob disease diagnostic. Mol Neurobiol. doi:10.1007/s12035-015-9167-5

28. Sanchez-Juan P, Sánchez-Valle R, Green A et al (2007) Influence of timing on CSF tests value for Creutzfeldt-Jakob disease diagnosis. J Neurol 254:901-906. doi:10.1007/s00415-006-0472-9

29. Llorens F, Schmitz M, Karch A et al (2015c) Comparative analysis of cerebrospinal fluid biomarkers in the differential diagnosis of neurodegenerative dementia. Alzheimers Dement:1-13. doi:10. 1016/j.jalz.2015.10.009 
30. Van Everbroeck B, Quoilin S, Boons J et al (2003) A prospective study of CSF markers in 250 patients with possible CreutzfeldtJakob disease. J Neurol Neurosurg Psychiatry 74:1210-1214. doi: 10.1136/jnnp.74.9.1210

31. Siman R, Giovannone N, Toraskar N et al (2011) Evidence that a panel of neurodegeneration biomarkers predicts vasospasm, infarction, and outcome in aneurysmal subarachnoid hemorrhage. PLoS One. doi:10.1371/journal.pone.0028938

32. Lemstra AW, van Meegen MT, Vreyling J et al (2000) 14-3-3 testing in diagnosing Creutzfeldt-Jakob disease: a prospective study in 112 patients. Neurology 55:514-516. doi:10.1212/WNL.55.4.514

33. Schoonenboom NSM, Reesink FE, Verwey NA et al (2012) Cerebrospinal fluid markers for differential dementia diagnosis in a large memory clinic cohort. Neurology 78:47-54. doi:10.1212/ WNL.0b013e31823ed0f0

34. Kaerst L, Kuhlmann A, Wedekind D et al (2013) Cerebrospinal fluid biomarkers in Alzheimer's disease, vascular dementia and ischemic stroke patients: a critical analysis. J Neurol 260:27222727. doi:10.1007/s00415-013-7047-3

35. del Campo M, Mollenhauer B, Bertolotto A et al (2012) Recommendations to standardize preanalytical confounding factors in Alzheimer's and Parkinson's disease cerebrospinal fluid biomarkers: an update. Biomark Med 6:419-430. doi:10.2217/ bmm.12.46

36. Kruse N, Mollenhauer B (2015) Validation of a commercially available enzyme-linked immunoabsorbent assay for the quantification of human ??-synuclein in cerebrospinal fluid. J Immunol Methods 426:70-75. doi:10.1016/j.jim.2015.08.003

37. Barbour R, Kling K, Anderson JP et al (2008) Red blood cells are the major source of alpha-synuclein in blood. Neurodegener Dis 5: 55-59. doi:10.1159/000112832

38. Llorens F, Karch A, Golanska E et al (2017) Cerebrospinal fluid biomarker-based diagnosis of sporadic Creutzfeldt-Jakob disease: a validation study for previously established cutoffs. Dement Geriatr Cogn Disord 43:71-80. doi:10.1159/000454802 